\section{MACROMOLECULES \\ Polyacids Pursued}

from our Molecular Biology Correspondent A DOMINANT attribute of biological macromolecules, in particular the nucleic acids, is their polyelectrolyte character, which in large measure determines their structure and interactions. One of the related problems which has been gnawed at, worried and shaken by any number of physical chemists is the nature of the all-pervading interaction of nucleic acids with magnesium. In a recent study, Sander and Ts'o (J. Mol. Biol., 55, 1 ; 1971) have taken advantage of the ion-selective membranes developed in recent years to reinvestigate the binding equilibria between magnesium and a series of nucleic acids and synthetic polynucleotides. The binding isotherms are complicated, indicating an effective heterogeneity of binding sites. Sander and Ts'o, making the simplest assumption-that of site binding, that is to say association of each ion with an individual charged site on the macromolecule-express the data in terms of the conventional binding plots (Scatchard equation) modified by an electrostatic interaction term, and extract an apparent binding constant for each species. The strongest binding is found in tRNA - and it will be recalled that the functional "native" state was found by Fresco and his colleagues to be characterized by the tight binding of one or two magnesium ions-followed by various synthetic polynucleotides in double-helical and single-stranded form and DNA and denatured DNA in no very revealing order. The strong binding and high interaction between sites in tRNA is surmised to be related to a compact, globular type of structure, which is not present in the other species examined.

The binding of magnesium to ribosomes, which is of course a prerequisite for structural integrity and biological function, has been studied by Walters and van Os (Biopolymers, 10, 11; 1971). Binding plots for yeast ribosomes showed prominent inflexions, which were present no matter what the concentration of sodium ions. The data can be fitted in terms of theory for the interaction of charged sites spread over a spherical body. The theory indicates that there should be little effect of subunit dissociation, which occurs within the range of the binding curves, on the electrostatic free energy, and this dissociation can therefore be disregarded. The interaction is again treated as site binding, and the stronger and weaker sets of sites are found to be in the ratio of $1.5: 1$.

The counter-ion layer that forms around a polyelectrolyte is strikingly reflected in the low-angle X-ray scatter- ing envelopes from DNA solutions, determined by Bram and Beeman (J. Mol . Biol., 55, 311; 1971). The results can be used to determine the apparent axial radius of gyration, and plots relating this parameter and the scattering angle show two limbs, reflecting the existence of two distinguishable radii of gyration. One of these corresponds to a mass: length ratio corresponding with remarkable precision to the expected value for the B-form of DNA.

Of greater novelty, however, is the second radius of gyration, which implies that the molecule can be represented as two concentric cylinders, the inner one of very high electron density. The outer shell is then surmised to consist of counterions, and possibly, Bram and Beeman suggest, water compressed by electrostriction. When the supporting electrolyte is sodium chloride the counterion shell is $20 \AA$ thick, and it increases as expected when the larger caesium counterions are substituted. The results of earlier workers, for a number of possible reasons, which are analysed by Bram and Beeman, failed to resolve this outer shell. The counterions are thus in the form of a Donnan sheath around the polyelectrolyte, and there is, as expected, no evidence of site binding. It would be of no little interest to examine DNA in the presence of magnesium ions.

A study of counterion interactions with a simple polyelectrolyte, polyvinylsulphonic acid, though without any direct biological orientation, deserves wide attention, both for its aesthetic value and the new directions that it may suggest to the physical biochemist. Taha and Morawetz (J.Amer.
Chem. Soc., 93, 829; 1971) have studied the fluorescence of uranyl ions as a function of the concentration of polyelectrolyte in the solution. The cations congregate in the domain of the polyacid and the fluorescence drops in consequence of self-quenching. In a $p \mathrm{H}$ range where the $\mathrm{UO}_{2}^{2+}$ ion is in equilibrium with polynuclear complexes $\left(\mathrm{U}_{2} \mathrm{O}_{5}{ }^{2+}\right.$ and higher $)$, rather different spectral changes occur in consequence of the preferential concentration of the mononuclear complex around the polyacid.

When a new quenching species, the ferrous ion, is introduced some striking effects are observed. At low ferrous ion concentration there is a dramatic drop in fluorescence, altogether different from the smooth progressive decline that is observed in the absence of a polyelectrolyte. This results from the confluence of the two anion species in the polyacid domain. Addition of more ferrous ions causes the displacement of the uranyl from the polyacid, and the fluorescence again rises to a maximum, after which quenching in the free solution supervenes and causes it again to fall. As Taha and Morawetz now point out, it is readily shown that such a profile of fluorescent intensity against quencher concentration can only be obtained if there are bound and unbound fractions of uranyl ions, which make separate contributions, and is incompatible with rapid exchange of counterions within the microsecond or so lifetime of the excited state of the uranyl. Thus, as expected, the sticking time of a counterion, which is treated in terms of site-binding, is much longer than this.

\title{
Transformations and Rotations
}

Atwater recently considered the relativistic treatments which are possible for rotating bodies and came to the conclusion that the application of special relativity could lead to impossible conclusions (Nature, 228, 272 ; 1970). In next week's Nature Physical Science, Marsh and Noonan separately take issue with him about the way in which he applies the Lorentz transformations to his example of a rotating disk.

It was pointed out by Atwater that a pattern of stripes painted on the edges of alternate octants of a transparent disk--which is then rotated at a high speed-would be Lorentz contracted in such a way that the stripes appear not to be uniformly distributed around the disk circumference. This is plainly unacceptable because of the inherent symmetry of a disk. Marsh suggests that it is anyway impossible to make use of the Lorentz transformation in the case of the periphery of a rotat- ing disk because the velocities of different points on the perimeter are not collinear. $\mathrm{He}$ also argues that, quite apart from kinematic arguments, the problems of the behaviour of rotating solids at speeds near to the speed of light have to be resolved before any definite conclusions can be reached about transformations involving rotating coordinate frames.

The possible effects of the stress created in a rotating solid are also emphasized by Noonan in his suggestion that the peculiarly unsymmetric situation, which, according to Atwater, is a necessary consequence of applying special relativity, could in fact be realized if the material were to crack under stress. Atwater's reply, to Noonan in particular, contains the very reasonable observation that the laws of physics must not depend on the intrinsic properties of materials. He also reemphasizes his original contention that an anomalous situation does exist. 